\title{
Dengue-2 infection and the induction of apoptosis in human primary monocytes
}

\author{
Amanda Torrentes-Carvalho, Elzinandes L Azeredo, Sonia RI Reis, Alessandro S Miranda, \\ Mariana Gandini, Luciana S Barbosa, Claire F Kubelka/+
}

Laboratório de Imunologia Viral, Instituto Oswaldo Cruz-Fiocruz, Av. Brasil 4365, 21045-900 Rio de Janeiro, RJ, Brasil

\begin{abstract}
Monocytes/macrophages are important targets for dengue virus (DENV) replication; they induce inflammatory mediators and are sources of viral dissemination in the initial phase of the disease. Apoptosis is an active process of cellular destruction genetically regulated, in which a complex enzymatic pathway is activated and may be trigged by many viral infections. Since the mechanisms of apoptotic induction in DENV-infected target cells are not yet defined, we investigated the virus-cell interaction using a model of primary human monocyte infection with DENV-2 with the aim of identifying apoptotic markers. Cultures analyzed by flow cytometry and confocal microscopy yielded DENV antigen positive cells with rates that peaked at the second day post infection (p.i.), decayed afterwards and produced the apoptosis-related cytokines TNF- $\alpha$ and IL-10. Phosphatidylserine, an early marker for apoptosis, was increased at the cell surface and the Fas death receptor was upregulated at the second day p.i. at significantly higher rates in $D E N V$ infected cell cultures than controls. However, no detectable changes were observed in the expression of the anti-apoptotic protein $\mathrm{Bcl}-2$ in infected cultures. Our data support virus modulation of extrinsic apoptotic factors in the in vitro model of human monocyte DENV-2 infection. DENV may be interfering in activation and death mechanisms by inducing apoptosis in target cells.
\end{abstract}

Key words: dengue - apoptosis - monocytes - human

The dengue fever emergency has become one of the most dramatic public health issues in Latin America. Epidemics are presenting increased severity and frequency and are continuously expanding geographically (Gubler 2002). In Brazil, the disease is endemic and during the last five years the region has accounted for $70 \%$ reported cases in the Americas (Nogueira et al. 2007). Infection with the Dengue virus (DENV) causes a disease spectrum ranging from asymptomatic to shock and hemorrhages, with a risk of lethality. Acute vascular permeability may be followed by abnormal vascular homeostasis. Clinical manifestations include plasmatic leakage, bleeding tendency and liver commitment followed by mild seric transaminase elevation. The physiopathology of the disease may be explained as result of infected monocyte activation by the production of factors that act to increase the vascular permeability and disturb the coagulation system. Pleural effusion, ascites and hemoconcentration are signs of intravascular volume loss. Such factors promote rapid progression to shock (Gibbons \& Vaughn 2002).

Mononuclear phagocytes are considered the main targets for viral replication (Jessie et al. 2004, NevesSouza et al. 2005). Cells such as B lymphocytes, monocytes, hepatocytes and dendritic cells are described as

Financial support: IOC-FIOCRUZ, DECICT/ CNPq, FAPERJ, ICGEB + Corresponding author: claire@ioc.fiocruz.br

Received 28 June 2009

Accepted 19 October 2009 potential virus targets and may undergo programmed cell death when infected in culture. Reports show apoptotic induction in DENV infected endothelial cells elevates IL-8 and RANTES production, which are chemokines related to inflammatory processes during cell migration (Avirutnan et al. 1998).

Monocytes/macrophages and dendritic cells are responsible for the dissemination of the virus after its entrance into the dermis by mosquito bite (Kou et al. 2008). Monocytes and $\mathrm{T}$ lymphocytes are activated during human infection, producing several pro-inflammatory mediators and may have amplified activation during secondary infection to become sources of intense cytokine production. Several investigations have followed patients during acute infection to monitor their plasma cytokine content and found that TNF- $\alpha$ is among the first detected cytokines in patients as well as in peripheral blood mononuclear cell (PBMC) cultures (Bethell et al. 1998, Braga et al. 2001, Reis et al. 2007). In general, these mediators may promote platelet and endothelial activation, culminating in thrombocytopenia, vascular permeability, hypotension and shock, as observed in severe cases of dengue (Fink et al. 2006). The TNF superfamily cytokines mediate their effects by activating transcription factors, including NF- $\kappa B$, which culminate in apoptotic processes and/or cellular proliferation (Karin \& Lin 2002).

Programmed cell death is an essential event during normal life in addition to being involved in pathophysiological processes that trigger disease (Lemasters 2005). Apoptosis consists of a controlled process of cell death that is orchestrated by a cascade of biochemical events, leading to morphologic changes and eventually to cell 
death (Bruchhaus et al. 2007). It is a physiologic mechanism that may exert pathogenic effects as well. Cells with damaged DNA can be removed without harm via the activation of a complex enzymatic chain of intracellular signaling systems that lead to the destruction of components that are essential to cell survival. These events occur in response to a variety of signals and stimuli, both internal and external, including growth factor deficiency, hormones, cytokines and suicidal cells that activate death receptors and endogenous proteases (Lemasters 2005). During the apoptotic process, changes in the cellular membrane expose phosphatidylserine to the external cell surface; subsequently, there is cleavage of the DNA double helix by endogenous endonucleases, among other changes (Vermes et al. 1995, Merchant et al. 2001).

Apoptosis is usually induced by an extrinsic signal transduction triggered by a death receptor such as TNF receptor (TNF-RI), TRAIL-RI/II and/or Fas (CD95/ APO-1) binding to TNF- $\alpha$, TRAIL or Fas ligand. After signaling, an enzymatic cascade leads to the activation of a series of cysteinyl aspartate proteases known as caspases (Feig \& Peter 2007) and then to cell degredation. However, the apoptotic process can be triggered by endogenous events as well. It is well-known that mitochondria play a central role in mediating intrinsic apoptotic signals. Changes in the external membrane mitochondrial potential and the production of reactive oxygen species are related to initial apoptotic events. When death signals reach the mitochondria, they lead to a collapse in the membrane potential that results in organelle rupture and release of pro-apoptotic proteins to cytoplasm, inducing caspase activation (Loeffler \& Kroemer 2000, Gupta 2003). The mitochondrial apoptotic program is also regulated by several Bcl-2 family members that are known to have an active role in inducting/inhibiting cell death (Gross 2001, Dejean et al. 2006).

DENV may be inducing apoptosis and disseminating its viral progenies to neighbor cells through phagocytized apoptotic bodies; alternatively, it may be acting to inhibit cell death in order to ensure virus survival and replication. Considering that phagocytosis of apoptotic cells is essential in preventing a deregulated inflammatory process and inducing specific immunological responses, the induction of apoptosis may be an attempt by the host immune system to limit the extent of the infection. Since monocytes/macrophages represent the main targets for infection by DENV and have an essential role in developing disease severity, it would be extremely useful to reveal processes that modulate the virus-cell interaction effect, with an emphasis on cell death mechanisms. To that end, we here studied an in vitro infection model in which primary human monocytes are infected with DENV-2, produce TNF- $\alpha$, IL-10 and are expressing apoptotic markers such as phosphatidylserine exposition and Fas upregulation, indicating that apoptotic processes are occurring.

\section{MATERIALS AND METHODS}

Virus strain, cell cultures, virus stock preparation and titration - DENV, serotype 2, strain 16681 was provided by Dr. SB Halstead (Naval Medical Research Cen- ter, USA). For virus stock preparation, an Aedes albopictus C6/36 cell clone was grown as a monolayer culture, as previously described by Reis et al. (2007). Virus stock was titrated by serial dilution cultures in microtiter plates and detected by immunofluorescence, as previously described (Miagostovich et al. 1993). Virus titer was calculated as $50 \%$ tissue culture infectious dose $\left(\mathrm{TCID}_{50} / \mathrm{mL}\right)$. The inactivated virus was prepared by incubating the inoculum for $30 \mathrm{~min} / 56^{\circ} \mathrm{C}$. Titrated virus stock was used in culture at a final concentration of $1.37 \times 10^{8} \mathrm{TCID}_{50} / \mathrm{mL}$.

In vitro purification of $P B M C$ - Human peripheral blood was obtained from the Hospital Universitário Clementino Fraga, Rio de Janeiro. PBMCs were isolated from healthy donors' buffy coats by centrifugation on a density gradient (350 g/30 min in Ficoll-Paque Plus, Amersham Biosciences Corp) according to standard procedures. Cell suspensions ( 1 × $10^{6}$ cells $\left./ \mathrm{mL}\right)$ in RPMI 1640 medium containing $200 \mathrm{mM}$ glutamine, $100 \mathrm{U} / \mathrm{mL}$ penicillin and $10 \mathrm{mg} / \mathrm{mL}$ streptomycin, were incubated at $37^{\circ} \mathrm{C}$ under humid atmosphere with 5\% CO2 and allowed to adhere on uncoated polystyrene flasks $\left(150 \mathrm{~cm}^{3}\right)$ during 90 min for monocyte enrichment. Non-adherent cells were gently removed by washing after which the adherent cells were detached by mechanical cell harvesting with cell scrapers in cold cell culture medium. Cell viability was verified in culture by Trypan blue exclusion and presented $\geq 95 \%$ viability. Enriched monocytes were suspended in RPMI 1640 medium supplemented with $10 \%$ FCS and seeded at $1 \times 10^{6}$ cells $/ \mathrm{mL}$ on 24 -well plates or 16-well cell culture chambers (Labtech, Nunc).

Infection of adherent PBMCs - After an overnight incubation, cell cultures were infected using a DENV-2 diluted inoculum $(300 \mu \mathrm{L})$, inactivated virus or maintained with culture medium. After $2 \mathrm{~h}$ incubation for adsorption, culture supernatants were replaced with medium containing 2\% FCS and further incubated for 24-120 h. Wells were set in triplicates for each different parameter in culture. After $48 \mathrm{~h}$, supernatants were collected and stocked at $-20^{\circ} \mathrm{C}$ until cytokine measurement and cells were recovered for viral antigen (Ag), co-stimulatory, intrinsic and extrinsic apoptotic marker determination by flow cytometry.

Determination of viral Ag and specific co-stimulatory marker by flow cytometry in human monocytes - Cells were recovered by scratching with a plastic microtip using cold medium and were set at $1 \times 10^{6}$ cells/microtube; they were then centrifuged (350 g, $5 \mathrm{~min}$ ) and washed once with $1 \mathrm{~mL}$ PBS pH 7.4 with 2\% FCS and 0,01\% $\mathrm{NaN}_{3}$. Single or double labeling of infected and uninfected adherent PBMCs was performed. For surface labeling, cells were blocked with 5\% inactivated plasma in PBS/ BSA at $4^{\circ} \mathrm{C} / 30$ min and incubated with FITC or Cy5.5labelled antibodies against CD14 (Southern Biotheck), a specific molecule for monocyte population. Matching isotype antibodies were used as a negative control. Intracellular staining after infection was performed as previously described (Neves-Souza et al. 2005), with slight modifications. Briefly, cells were fixed with $0.5 \mathrm{~mL}$ cold paraformaldehyde at 2\% in PBS for 10 min and, after 
centrifugation, membrane permeabilization was carried out with $1 \mathrm{~mL} 0.1 \%$ saponine in PBS with FCS and $\mathrm{NaN}_{3}$. Cell surface unspecific receptors were blocked with $5 \%$ inactivated human plasma in PBS/BSA at $4^{\circ} \mathrm{C} / 30 \mathrm{~min}$. Primary monoclonal antibodies reactive to Dengue Complex (Chemicon) or isotype anti-mouse IgG2a diluted in PBS with saponine, FCS and $\mathrm{NaN}_{3}$ were added to cells for a 60 min incubation. Cells were washed once with $1 \mathrm{~mL}$ PBS containing FCS and $\mathrm{NaN}_{3}$ and further incubated with a secondary anti-mouse IgG1 labeled with Alexa 488 (BD Pharmingen) for 30 min. Alternatively, for double staining with CD14 and DENV, cells were first surface labeled with PE-labeled antibodies to CD14 (DAKO) and then intracellularly labeled with monoclonal antibody to Dengue Complex-Alexa 647 (coupled with Molecular Probes labeling kit). Cells were then acquired (5,000 events for gated monocytes) on a FACSCalibur flow cytometer (Beckon \& Dickinson). Analysis was performed using FlowJo Software (TreeStar Inc).

Assessment of apoptosis - Apoptotic detection assays were carried out by surface labeling with the $\mathrm{Ca}^{2+}$ dependent phosphatidylserine-binding protein annexin V. Uninfected and infected cells were recovered by scratching with plastic microtips using cold medium and were set at $1 \times 10^{6} /$ microtube; they were then centrifuged (350 g, $5 \mathrm{~min}$ ) and washed once with PBS 1X. Cells were labeled by incubation with Annexin V-FITC and propidium iodide (PI) in a binding buffer (Apoptosis detection Kit I \# 556547, BD Pharmingen) for 15 min at RT in the dark, according to the manufacturer's instruction. For extrinsic (surface) apoptotic molecules labeling, cells were blocked and incubated with FITC-labeled antibodies to Fas (Southern Bioteck), a death receptor. Intracellular staining was performed for detection of Bcl-2, an intrinsic anti-apoptotic protein, using PE-labeled antibodies to Bcl-2 (Caltag) as described above. Specific isotypes were used as negative controls. For an apoptotic positive control, culture wells were treated with Brefeldin A (Sigma), a fungal macrocyclic lactone that is a potent inhibitor of membrane trafficking and vesicular transport and which therefore induces apoptosis when incubated with cultures at low concentrations for long time periods (Shao et al. 1996, Guo et al. 1998). Stained cells were acquired (5,000 for gated monocytes) on a FACSCalibur flow cytometer. Analysis was performed using FlowJo Software.

Confocal microscopy - Cells were isolated and cultured in Labtech microchambers (Nunc). Two days after infection, as described above, supernatants were recovered and adherent cells were washed once with PBS $1 \mathrm{X}$ and fixed with $0.05 \mathrm{~mL}$ cold paraformaldehyde at $2 \%$ and Triton $0.2 \%$ (Sigma) at $4^{\circ} \mathrm{C} / 30 \mathrm{~min}$. After fixation, wells were washed with PBS/BSA and blocked with 5\% inactivated plasma in $\mathrm{PBS} / \mathrm{saponine}$ at $4^{\circ} \mathrm{C} / 30 \mathrm{~min}$. Subsequently, cells were incubated with Dengue Complex-reactive primary monoclonal antibodies or isotype anti-mouse IgG2a (Chemicon) for $37^{\circ} \mathrm{C} / 60 \mathrm{~min}$ in a humid chamber. Cells were washed twice with PBS/BSA and further labelled with a secondary anti-mouse IgG1 coupled to Alexa 488 (BD Pharmingen) for $37^{\circ} \mathrm{C} / 60 \mathrm{~min}$ in a humid chamber. The microchambers were washed with distillated water and the chambers were removed from slides. Slides were dried and mounted in glycerol with $2 \%$ anti-fading agent. Cells were observed under a fluorescence microscope LSM510-META (Zeiss) using excitation/emission $(488 \mathrm{~nm} / 540 \mathrm{~nm}$ - green fluorescence dye) for DENV. Digital pictures of nuclei and virus were obtained for each stain using a CCD camera.

Ethics - Procedures performed during this work were approved by the Ethical Committee of the Fundação Oswaldo Cruz, Brazilian Health Ministry (recognized by the Brazilian National Ethical Committee) (111/00).

Statistical analyses - Data were first tested for normality with the GraphPad Prism version 5 for Windows GraphPad Software (http://www.graphpad.com) in order to determine the significance of differences in dengue Ag, Annexin V and Fas death receptor positive cell numbers under several culture treatment conditions. Data values passed the Kolmogorow-Smirnov normality test and were evaluated for significance using the one-way ANOVA (more than two groups). Altered parameters were considered significant at $\mathrm{p}<0.05$.

\section{RESULTS}

DENV Ags are expressed after monocyte infection; proinflammatory cytokine TNF-a production peaked at two days and IL-10 at three days post infection (p.i.) Monocytes infected with DENV-2 (strain 16681) were cultured for five days in order to evaluate cell infection and cytokine production. Both DENV intracellular Ag and secreted cytokines present in the cell culture supernatant were determined daily. Cytoplasmatic virus localization was observed by confocal microscopy with immunofluorescent labeling (Fig. 1A). Infected cells are $\mathrm{CD}_{14}{ }^{+}$monocytes as represented by contour plots during flow cytometry analysis within the monocyte gate (Fig. 1B). Increased DENV $\mathrm{Ag}^{+}$cell rates were detected by flow cytometry until the second day p.i., when peak values were reached. After this time point, $\mathrm{DENV} \mathrm{Ag}^{+}$ cell percentages decreased (Fig. 1C). Monocytes from 12 PBMC healthy donors showed mean virus infection percentage of $40 \pm 4 \%$ on the second day p.i. with a minimum of $18 \%$ and a maximum of $58 \%$ DENV $\mathrm{Ag}^{+}$cells.

TNF- $\alpha$ is a pro-inflammatory cytokine that followed the same kinetic pattern as cell infection, with maximum levels at day two and a subsequent gradual decay from 3-5 days after infection (Fig. 2). IL-10 is an anti-inflammatory cytokine that may be induced by TNF- $\alpha$ as a regulatory feedback mechanism and reached its peak a day later. Both are detected in cell culture supernatants and are most likely produced as a result of cell activation by the virus and stay in culture longer than viral Ags detected intracellularly. Most experiments performed showed that intracellular virus was already high by the first day, peaked at the second day and then decayed abruptly and from the third day onwards was either undetected or detected at low rates. In contrast, cytokines displayed statistically significant differences in their levels for 3-5 days.

\section{Changes related to programed cell death in mono- cytes infected with DENV-2}

Membrane changes exposing phosphatidylserine occur on infected monocytes - In order to evaluate if DENV 
A
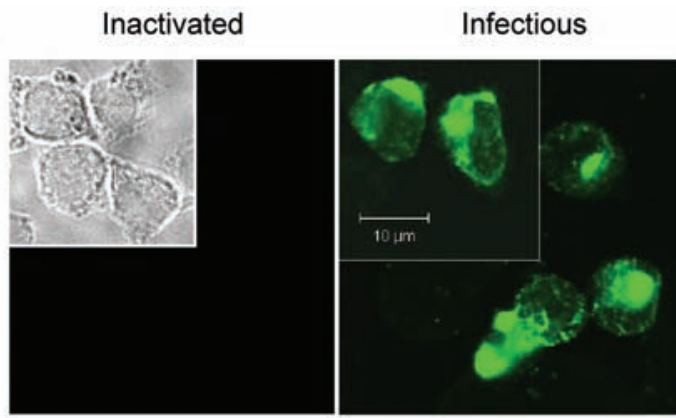

B

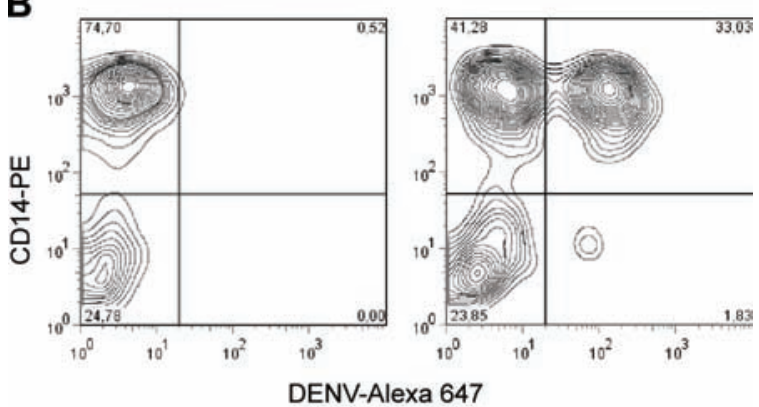

C

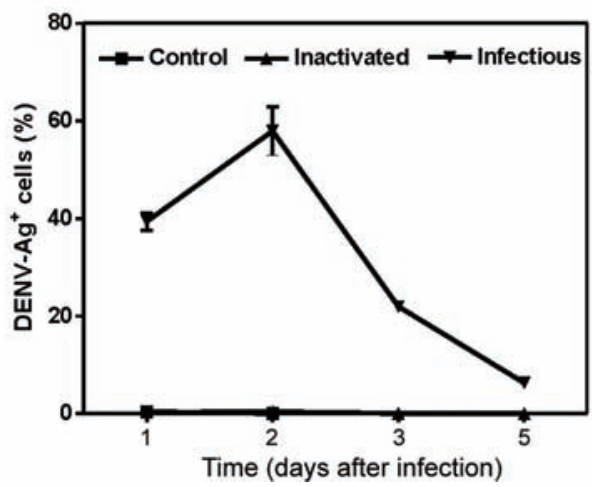

Fig. 1: Dengue virus (DENV)- antigen (Ag) detection in human monocytes after in vitro DENV-2 infection: monocyte-enriched human peripheral blood mononuclear cell (PBMC) were incubated for 2-5 days with infectious DENV-2 (strain 16681) and negative controls (heat-inactivated virus and culture medium). Cells were labeled with antibody to DENV-complex and anti-mouse IgG Alexa-488 or -647. DENV Ag was detected in infected monocytes by (A) confocal microscopy in two different fields or by (B) flow cytometry observed in contour plots. Time dependent DENV Ag detection was determined by FACS in three different PBMC donors (C) (average \pm standard error is plotted).

was inducing apoptosis in human monocytes, we searched for an early marker of cell death, the display of phosphatidylserine at the cell membrane, by labeling with annexin $\mathrm{V}$ and PI. The presence of annexin V without PI labeling is used to identify apoptotic and autophagic cells at early stages of cell death, when annexin $\mathrm{V}$ binding is exclusive. Double-labeling occurs at later stages of apoptosis and in subsequent necrotic processes. Brefeldin A, a lactone antibiotic produced by fungal organisms that induces apoptosis, was added to cell cultures as positive control.

Two days after infection of monocytes with DENV-2, cells were recovered for flow cytometry analysis. Fig. 3A
A

TNF- $\alpha$

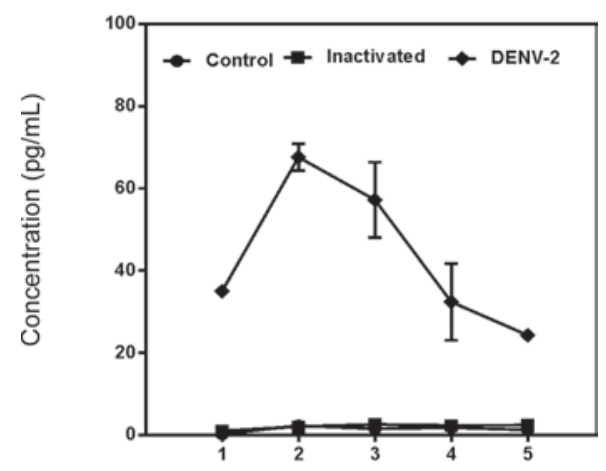

B

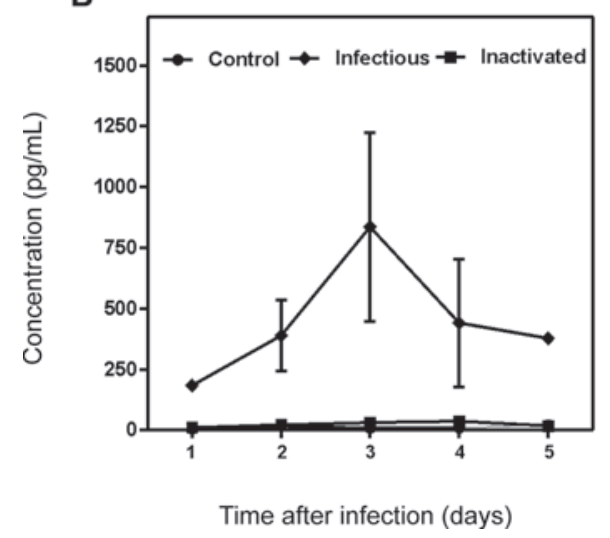

Fig. 2: proinflammatory cytokine TNF- $\alpha$ and Il-10 production after monocyte infection by Dengue virus (DENV)-2: monocyte-enriched human peripheral blood mononuclear cell (PBMC) was infected with DENV-2 (strain 16681). Medium and inactivated DENV were used as controls and at 1-5 day incubation time cell culture supernatants were tested for TNF- $\alpha$ production by ELISA. Three PBMC donors were assayed.

shows contour plots representing annexin V and PI labeling on monocytes incubated with cell culture medium, inactivated virus, infectious DENV-2 or brefeldin A. Infected cells presented an increase in single-labeled annexin $\mathrm{V}^{+}$cells as compared to cultures incubated with inactivated virus or medium. No significant differences were observed in the frequency of cells that underwent apoptosis followed by necrosis (annexin- $\mathrm{V}^{+} \mathrm{PI}^{+}$) when inactivated was compared to infectious virus. Cells incubated only with inactivated virus displayed equivalent percentages as those incubated with medium, indicating that physiological culture conditions are permitting cells to die spontaneously or recovery procedures may damage some cells. Nevertheless, the virus enhanced the apoptotic process that was occurring in culture. Data from five different PBMC donors for annexin $\mathrm{V}^{+} \mathrm{PI}^{-}$cells are plotted in Fig. 4A, showing the significant difference between cultures exposed to infectious DENV-2 and controls. Differences in early apoptotic markers between infected and uninfected cells were not significant until $48 \mathrm{~h}$.

Fas receptor expression is detected on infected monocytes - The Fas cell surface receptor (CD95) can be stim- 

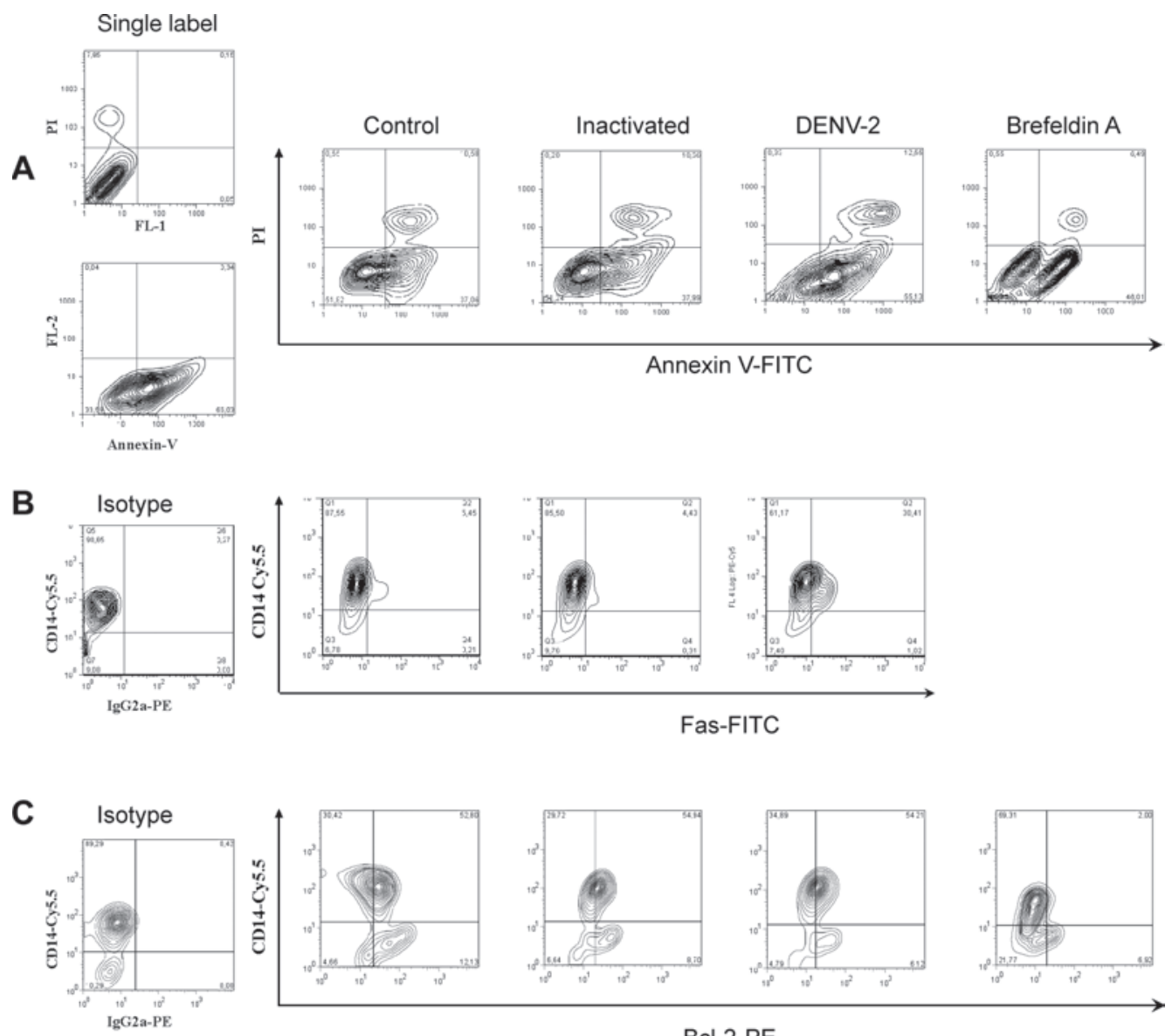

Fig. 3: contour plots from apoptotic cells after monocyte infection by dengue virus (DENV)-2: monocyte-enriched human peripheral blood mononuclear cell (PBMC) was incubated for two days with infectious DENV-2 (strain 16681), negative controls (cell culture medium or heatinactivated virus) or brefeldin A. Cells were labeled with (A) FITC-labeled annexin V to detect early apoptotic cells and with propidium iodide (PI) to detect necrosis, (B) CD14-Cy5 plotted against anti-Fas-FITC (FCS) and (C) anti-CD14-Cy5 plotted against anti-Bcl-2-PE. Monocyte gated cells were analyzed. Isotype/single labeling is shown at the left side.

ulated by agonists or the natural ligand FasL, recruiting proteins with distinct functions such as cytosolic adaptor proteins that ultimately bind to the initiating caspase (caspase 8) and trigger apoptosis (Limjindaporn et al. 2007). DENV-2 infected monocytes were also analyzed in order to detect the Fas expression on cell surface. By the first day after infection, $\mathrm{Fas}^{+}$cells were already more common in DENV-2 exposed cells than in controls. At two days after infection, Fas $^{+}$cell frequencies were significantly increased in the presence of the infectious virus, as detected in six different PBMC donors (Fig. 4B).

$\mathrm{BCl}-2$ oncoprotein expression is not altered in infected monocytes - The proto-oncogene Bcl-2 works as an important repressor of programmed cell death. We investigated whether DENV-2 infected monocytes, apparently more susceptible to death, might have altered $\mathrm{Bcl}-2$ expression and therefore an altered degree of inhibition of apoptosis. We detected $\mathrm{Bcl}-2$ by labeling monocytes from 1-5 days after DENV-2 infection and analysed the cells by flow cytometry (data not shown). $\mathrm{Bcl}-2$ expression remained unchanged during this peri- od, although alternative treatment with brefeldin A was able to downregulate this protein, as can be observed in Fig. 3C (showing data from the first $48 \mathrm{~h}$ ). In order to ascertain whether cells were infected or not with DENV, we performed double-labeling with antibodies directed against Bcl-2 and DENV. We detected double labeling in $39 \pm 4 \%$ cells at $24 \mathrm{~h}$ and $50 \pm 2 \%$ at $48 \mathrm{~h}$, in contrast to negative double label in controls $(<1 \%)$, indicating that the presence of the DENV-2 does not interfere with Bcl-2 expression; most infected cells are still expressing this protein and the apoptosic mechanisms appear to act independently from $\mathrm{Bcl}-2$ regulation.

\section{DISCUSSION}

Mononuclear phagocytes such as monocytes and dendritic cells are the main DENV target cells, both in vitro and in vivo and their interaction with the virus has been shown to be relevant to the host response to infection (Halstead \& O'Rourke 1977, Wu et al. 2000, Neves-Souza et al. 2005). After signaling pathway activation by viruses, these cells have upregulated surface activation molecules 

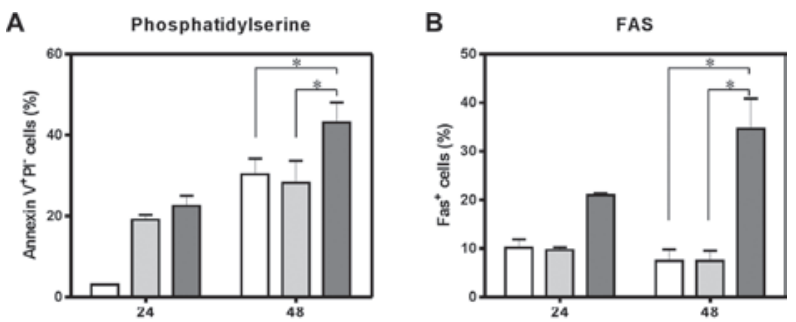

C

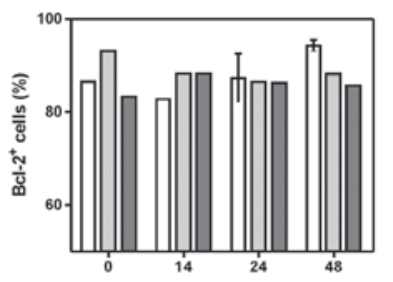

Time (hours after infection)

Fig. 4: Dengue virus (DENV)-2 infection enhanced apoptosis of human monocytes as observed by Fas and phosphatidilserine detection but no Bcl-2 change: monocyte-enriched human peripheral blood mononuclear cell (PBMC) was incubated for 14-48 h with infectious DENV-2 (strain 16681) or negative control (heat-inactivated virus or culture medium). Cells were labeled with (A) annexin V- FITC to detect early apoptotic cells and with propidium iodide (PI) to detect necrosis ( $\mathrm{n}=2$ at $24 \mathrm{~h} ; \mathrm{n}=6$ at $48 \mathrm{~h}$ ) and (B) anti-Fas-FITC ( $\mathrm{n}=1$ at 24 $\mathrm{h} ; \mathrm{n}=5$ at $48 \mathrm{~h}$ ) or (C) Bcl-2 PE ( $\mathrm{n}=4$ all time points). Monocyte gated cells were analyzed. Asterisks means $\mathrm{p}<0.05$ in Wilcoxon test.

and begin producing cytokines and chemokines. Modulation of the innate and adaptative immunity interface occurs as a result (Frankenberger et al. 1996, Bosch et al. 2002, Strauss-Ayali et al. 2007), likely influencing the protection and/or pathology onset during infection.

DENV replicates in human primary peripheral monocytes (Halstead \& O'Rourke 1977, Chen \& Wang 2002). We have previously infected human monocyte cultures with DENV-2 Asian strain 16681 (Sydow et al. 2000) and other DENV strains (Neves-Souza et al. 2005). Here we show a time-dependent detection of DENV Ags starting as early as the first day after infection, with a detection peak at $48 \mathrm{~h}$ and a subsequent decay. These results resemble other reports using this virus strain in dendritic cells, although peak virus production may be related not only to the strain type but also with the inoculum concentration (Cologna \& Rico-Hesse 2003). Intracellular virus detection was related to TNF- $\alpha$ extracellular production, with a maximum production at the second day. TNF- $\alpha$ is probably produced as a result of virus replication and remains in culture, being detected at significant levels at five days, by which point intracellular virus is found at low levels. It is likely that non-infected monocytes are activated and become important targets for TNF- $\alpha$ production at the later stage of infection.

Programmed cell death is an essential regulatory process in the immunological system. During immune responses to viral infections, apoptosis may occur as a direct pathogenic mechanism of viral escape, allowing apoptotic bodies containing virus to disseminate infec- tion to neighbor cells. Alternatively, apoptosis may be used as an immune mechanism to induce opposed effects, becoming deleterious to viral progenies and representing an appropriate response from host to limit viral replication (Servet-Delprat et al. 2000). Apoptosis can be either Ag-dependent, initiated by death receptor signaling and/or activating intracellular pathways, or it may occur by negligence when Ag-specific receptors are not stimulated. When the cellular microenvironment is depleted of survival factors, another type of programmed cell death occurs, a phenomenon known as authophagy (Lemasters 2005, Feig \& Peter 2007). Moreover, modulation of programmed cell death plays an important role in several pathologies related to viral infections such as AIDS and measles (Oyaizu \& Pahwa 1995, Servet-Delprat et al. 2000). DENV replication seems to be essential to induce both phagocytosis and apoptosis in human monocytes, as reported by Espina et al. (2003). However, these authors showed no quantitative evidence about apoptotic events. Considering that monocytes are among the main target cells for DENV, we investigated markers of programmed cell death during human primary monocyte infection, as well as the production of related cytokines.

During phagocytosis, the virus-cell interaction may lead to production of cytoplasmatic lysosomal components that can result in deleterious effects for both the virus and host cells. Labeling cells with annexin V, which binds to the exposed phosphatidylserine found on apoptotic cells and the simultaneous exclusion with propidium iodine evaluated by flow cytometry allowed us to quantify early apoptosis after two days of DENV infection in monocytes. This observation matches with the peak production of TNF- $\alpha$, which is a proinflammatory cytokine that plays an important role in the induction of apoptosis. We suggest that the maturation process and the cytokine production by these cells are activation pathways involved in apoptotic processes. We also observed that some monocytes cultured without infectious virus die, although at lower rates. It is possible that they are undergoing cell death processes by mechanisms such as authophagy if survival factors are not present at sufficient concentrations in cultures (Yu et al. 2006).

Among TNF family receptor series, the Fas receptor is related to the pathenogenesis of viral infection such as measles and hepatitis B (Servet-Delprat et al. 2000, Wang et al. 2004). Dendritic cells infected by measles virus present upregulated expression of Fas, becoming more susceptible to apoptosis mediated by the Fas/FasL system. Also, a protein from the DENV capsid induces apoptosis in HepG2 cells in a Fas-dependent fashion (Limjindaporn et al. 2007). Fas signaling induces IL-8, TNF- $\alpha$ and IL-1 $\beta$ production in mononuclear phagocytes, modulating pro-inflammatory responses by a caspase-dependent pathway (Park et al. 2003).

IL-10 is a potent anti-inflammatory cytokine. This factor triggers apoptosis in monocytes via CD95 system and monocytes undergoing spontaneous apoptosis in vitro become a potent source of IL-10 (Schmidt et al. 2000, Bzowska et al. 2002). Elevated Il-10 levels have 


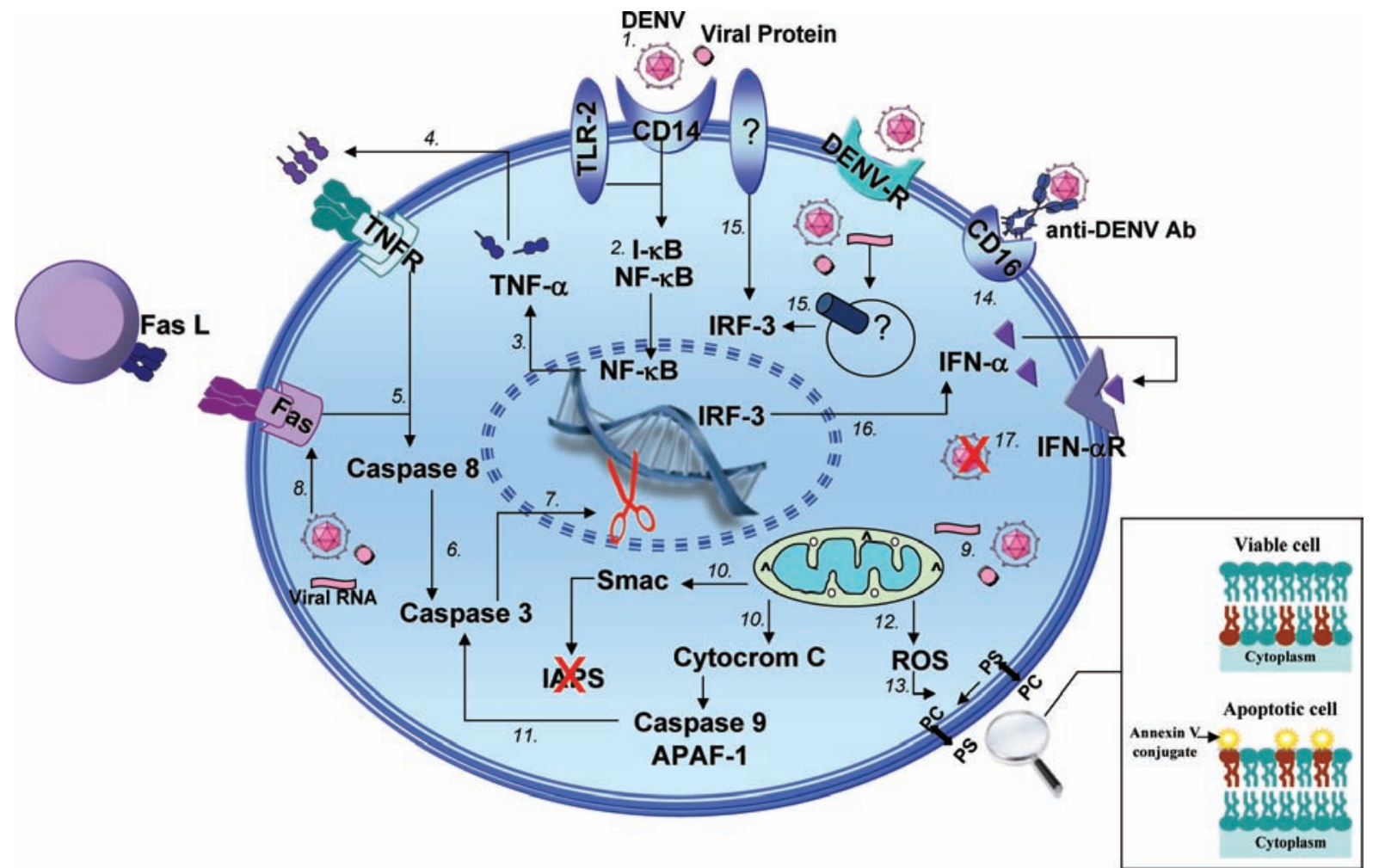

Fig. 5: hypothesis for signaling pathways involved in cell death and cellular activation during monocyte infection by dengue virus (DENV). After DENV inoculation by Aedes aegypti mosquito into the human host skin, the virus would infect one of the main target cells - circulating monocytes - through their co-receptors such as TLR-2 (CD14-TLR-2) or by other not yet known cellular receptor (1). The recognition and/or viral endocytosis mediated by these receptors would lead to the activation of transcription factors in charged of the cytokine induction and other mediators involved in viral clearance and in the survival/death from the infected cell. Among transcription factors, the I $\mathrm{B}-\mathrm{NF}-\kappa \mathrm{B}$ complex is dissociated, permitting the NF- $\mathrm{KB}$ translocation to the nucleus (2) and, consequently, inducing proinflammatory cytokine expression such as TNF- $\alpha$ (3). TNF- $\alpha$ interaction with its transmembrane receptors, mainly TNFR1, induces recruitment from another series of cytoplasmatic signaling proteins (4) that lead initially to caspase-8 activation (5). Within a sequence of well orientated events, the caspase-8 induces effector caspase-3 activation (6) that is involved in inhibition and/or activation of cytoplasmatic constituents in charged of maintaining cell survival (7). During infection it would be also possible to observe the increase in the Fas receptor expression (8), which interacts with its ligand - FasL, present in neighbor cells, triggers the apoptosis signaling cascade similarly to the TNF/TNFR pathway. The virus infection would also activate the intrinsic cell death pathway, in which mitochondria has a critical role (9). Indeed, several reports have been demonstrating that an alteration in mitochondrial membrane permeability promotes the organelle dysfunction (10) and secretion of apoptotic proteins that activate caspase-9, which in a sequence, activates caspase-3 (11). The mitochondrial membrane potential and permeability alterations lead to free radical secretion (12) that, e. g., would contribute to the cell death processes by inducing phosphatidylserine exposition on external membrane from the apoptotic cell, facilitating in this way, their recognition by phagocytic cells (13). The cell death activation may be associated with monocyte activation events, such as the increase from CD16 (FC $\gamma I I I)$, characteristic of monocytes with high proinflammatory capacity (14). On the other hand, the infection by DENV would induce the transcription factor IRF-3 (15), involved in the increase of anti-viral cytokines such as IFN- $\alpha$ (16). The effector pathway for type I IFNs would induce the anti-viral gene expression involved in the viral replication inhibition (17).

been detected in patients with dengue fever and often have been found to be associated with increased severity (Green et al. 1999, Azeredo et al. 2001).

We demonstrated here for the first time that human monocytes infected in vitro by DENV have upregulated Fas expression concomitant with the viral peak, indicating that programmed cell death induced by DENV in our study model may be influenced by this extrinsic apoptotic pathway. It is possible that this process leads to extracellular release of virus, facilitating neighbor cell infection, in addition to inducing simultaneously monocyte maturation. Fas and DENV Ag double cell labeling in DENV-2 infected cultures shows that Fas expression may occur in DENV-Ag positive and negative cells. DENV-Ag is detected in Fas-negative cells (data not shown). Although our data indicate that apoptosis is induced in monocytes during Fas expression, we cannot exclude the possibility that TNF- $\alpha$ /TNFR receptor, cytokines and/or other mechanisms are participating in apoptotic processes mediated by DENV. Azeredo at al. (2001) reported elevated circulating TNF- $\alpha$ and TNF soluble receptors in patients with acute dengue fever. The cytoplasmatic expression of the proto-oncogene Bcl-2 plays a role in increasing cell survival and has contrary effects to Fas during apoptosis (Boudet et al. 1996, Molica et al. 1996). However, no significant changes were detected with regard to $\mathrm{Bcl}-2$ expression in monocytes infected with DENV-2, in contrast to what we observed 
in CD8 positive T-lymphocytes from dengue patients, which presented low levels of Bcl-2 (Azeredo et al. 2006). The present data suggest that other anti-apoptotic proteins, such as $\mathrm{Bcl}-\mathrm{xL}$, might be negatively regulated during infection in monocytes.

According to our data and other relevant studies, we present here a model for DENV infection in monocytes in which complex interactions between the virus and host cell occur, leading to the activation of transcription factors, cytokines and enzymatic factors. These interactions may induce not only pathologic proinflammatory responses that influence the severity and progression of the disease, but also virus clearance and apoptotic pathways that could be controlling infection by anti-viral mechanisms [(Chen et al. 1999, Diamond et al. 2000, Bielefeldt-Ohmann et al. 2001, Grandvaux et al. 2002, Navarro-Sanchez et al. 2003, Neves-Souza et al. 2005, Azeredo et al. 2006, 2009, Seth et al. 2006, Chareonsirisuthigul et al. 2007, Reis et al. 2007, Durbin et al. 2008, Kou et al. 2008 (see details in Fig. 5)]. The present study may, therefore, contribute to a better understanding of the cell activation mechanisms triggered during DENV infections occurring in human target cells. Further investigations will be required in order to define in detail the apoptotic pathways involved in dengue fever and to determine whether or not there is a potential benefic role from apoptosis in the resolution of infection.

\section{ACKNOWLEDGMENTS}

To Bernardo M Pascarelli, by helping in confocal image analysis and uptake at the Confocal Microscopy Technological Platform, Fiocruz, and to Luzia Maria de Oliveira Pinto, for suggestions and encouragement. Amanda Torrentes de Carvalho was a fellowship from CNPq.

\section{REFERENCES}

Avirutnan P, Malasit P, Seliger B, Bhakdi S, Husmann M 1998. Dengue virus infection of human endothelial cells leads to chemokine production, complement activation and apoptosis. J Immunol 161: 6338-6346.

Azeredo EL, Neves-Souza PC, Alvarenga AR, Reis SRNI, TorrentesCarvalho A, Zagne SMO, Nogueira RMR, Oliveira-Pinto LM, Kubelka CF 2009. TLR-2, TLR-4 and CD16 are upregulated in human monocytes after dengue virus in vitro infection and are associated with mild dengue in patients, while HLA-DR is downregulated during severe dengue fever. Immunology, in press.

Azeredo EL, Zagne SMO, Alvarenga AR, Nogueira RMR, Kubelka CF, Oliveira-Pinto LM 2006. Activated peripheral lymphocytes with increased expression of cell adhesion molecules and cytotoxic markers are associated with dengue fever disease. Mem Inst Oswaldo Cruz 101: 437-449.

Azeredo EL, Zagne SM, Santiago MA, Gouvea AS, Santana AA, Neves-Souza PC, Nogueira RM, Miagostovich MP, Kubelka CF 2001. Characterisation of lymphocyte response and cytokine patterns in patients with dengue fever. Immunobiology 204: 494-507.

Bethell DB, Flobbe K, Cao XT, Day NP, Pham TP, Buurman WA, Cardosa MJ, White NJ, Kwiatkowski D 1998. Pathophysiologic and prognostic role of cytokines in dengue hemorrhagic fever. J Infect Dis 177: 778-782.

Bielefeldt-Ohmann H, Meyer M, Fitzpatrick DR, Mackenzie JS 2001. Dengue virus binding to human leukocyte cell lines: re- ceptor usage differs between cell types and virus strains. Virus Res 73: 81-89.

Bosch I, Xhaja K, Estevez L, Raines G, Melichar H, Warke RV, Fournier MV, Ennis FA, Rothman AL 2002. Increased production of interleukin-8 in primary human monocytes and in human epithelial and endothelial cell lines after dengue virus challenge. J Virol 76: 5588-5597.

Boudet F, Lecoeur H, Gougeon ML 1996. Apoptosis associated with ex vivo down-regulation of Bcl-2 and up-regulation of Fas in potential cytotoxic CD8 ${ }^{+} \mathrm{T}$ lymphocytes during HIV infection. J Immunol 156: 2282-2293.

Braga EL, Moura P, Pinto LM, Ignacio SR, Oliveira MJ, Cordeiro MT, Kubelka CF 2001. Detection of circulant tumor necrosis factoralpha, soluble tumor necrosis factor p75 and interferon-gamma in Brazilian patients with dengue fever and dengue hemorrhagic fever. Mem Inst Oswaldo Cruz 96: 229-232.

Bruchhaus I, Roeder T, Rennenberg A, Heussler VT 2007. Protozoan parasites: programmed cell death as a mechanism of parasitism. Trends Parasitol 23: 376-383.

Bzowska M, Guzik K, Barczyk K, Ernst M, Flad HD, Pryjma J 2002. Increased IL-10 production during spontaneous apoptosis of monocytes. Eur J Immunol 32: 2011-2020.

Chareonsirisuthigul T, Kalayanarooj S, Ubol S 2007. Dengue virus (DENV) antibody-dependent enhancement of infection upregulates the production of anti-inflammatory cytokines, but suppresses anti-DENV free radical and pro-inflammatory cytokine production in THP-1 cells. J Gen Virol 88: 365-375.

Chen YC, Wang SY 2002. Activation of terminally differentiated human monocytes/macrophages by dengue virus: productive infection, hierarchical production of innate cytokines and chemokines and the synergistic effect of lipopolysaccharide. J Virol 76: 9877-9887.

Chen YC, Wang SY, King CC 1999. Bacterial lipopolysaccharide inhibits dengue virus infection of primary human monocytes/ macrophages by blockade of virus entry via a CD14-dependent mechanism. $J$ Virol 73: 2650-2657.

Cologna R, Rico-Hesse R 2003. American genotype structures decrease dengue virus output from human monocytes and dendritic cells. J Virol 77: 3929-3938.

Dejean LM, Martinez-Caballero S, Manon S, Kinnally KW 2006. Regulation of the mitochondrial apoptosis-induced channel, MAC, by BCL-2 family proteins. Biochim Biophys Acta 1762: 191-201.

Diamond MS, Roberts TG, Edgil D, Lu B, Ernst J, Harris E 2000. Modulation of dengue virus infection in human cells by alpha, beta and gamma interferons. $J$ Virol 74: 4957-4966.

Durbin AP, Vargas MJ, Wanionek K, Hammond SN, Gordon A, Rocha C, Balmaseda A, Harris E 2008. Phenotyping of peripheral blood mononuclear cells during acute dengue illness demonstrates infection and increased activation of monocytes in severe cases compared to classic dengue fever. Virology 376: 429-435.

Espina LM, Valero NJ, Hernandez JM, Mosquera JA 2003. Increased apoptosis and expression of tumor necrosis factor-alpha caused by infection of cultured human monocytes with dengue virus. Am J Trop Med Hyg 68: 48-53.

Feig C, Peter ME 2007. How apoptosis got the immune system in shape. Eur J Immunol 37 (Suppl 1): S61-70.

Fink J, Gu F, Vasudevan SG 2006. Role of T cells, cytokines and antibody in dengue fever and dengue haemorrhagic fever. Rev Med Virol 16: 263-275.

Frankenberger M, Sternsdorf T, Pechumer H, Pforte A, Ziegler-Heitbrock HW 1996. Differential cytokine expression in human blood 
monocyte subpopulations: a polymerase chain reaction analysis. Blood 87: 373-377.

Gibbons RV, Vaughn DW 2002. Dengue: an escalating problem. BMJ 324: 1563-1566.

Grandvaux N, Ten Oever BR, Servant MJ, Hiscott J 2002. The interferon antiviral response: from viral invasion to evasion. Curr Opin Infect Dis 15: 259-267.

Green S, Vaughn DW, Kalayanarooj S, Nimmannitya S, Suntayakorn S, Nisalak A, Rothman AL, Ennis FA 1999. Elevated plasma interleukin-10 levels in acute dengue correlate with disease severity. J Med Virol 59: 329-334.

Gross A 2001. BCL-2 proteins: regulators of the mitochondrial apoptotic program. IUBMB Life 52: 231-236.

Gubler DJ 2002. The global emergence/resurgence of arboviral diseases as public health problems. Arch Med Res 33: 330-342.

Guo H, Tittle TV, Allen H, Maziarz RT 1998. Brefeldin A-mediated apoptosis requires the activation of caspases and is inhibited by Bcl-2. Exp Cell Res 245: 57-68.

Gupta S 2003. Molecular signaling in death receptor and mitochondrial pathways of apoptosis - Review. Int J Oncol 22: 15-20.

Halstead SB, O’Rourke EJ 1977. Dengue viruses and mononuclear phagocytes. I. Infection enhancement by non-neutralizing antibody. J Exp Med 146: 201-217.

Jessie K, Fong MY, Devi S, Lam SK, Wong KT 2004. Localization of dengue virus in naturally infected human tissues, by immunohistochemistry and in situ hybridization. J Infect Dis 189: 1411-1418.

Karin M, Lin A 2002. NF-kappaB at the crossroads of life and death. Nat Immunol 3: 221-227.

Kou Z, Quinn M, Chen H, Rodrigo WW, Rose RC, Schlesinger JJ, Jin X 2008. Monocytes, but not T or B cells, are the principal target cells for dengue virus (DV) infection among human peripheral blood mononuclear cells. J Med Virol 80: 134-146.

Lemasters JJ 2005. Dying a thousand deaths: redundant pathways from different organelles to apoptosis and necrosis. Gastroenterology 129: 351-360.

Limjindaporn T, Netsawang J, Noisakran S, Thiemmeca S, Wongwiwat W, Sudsaward S, Avirutnan P, Puttikhunt C, Kasinrerk W, Sriburi R, Sittisombut N, Yenchitsomanus PT, Malasit P 2007. Sensitization to Fas-mediated apoptosis by dengue virus capsid protein. Biochem Biophys Res Commun 362: 334-339.

Loeffler M, Kroemer G 2000. The mitochondrion in cell death control: certainties and incognita. Exp Cell Res 256: 19-26.

Merchant SH, Gonchoroff NJ, Hutchison RE 2001. Apoptotic index by Annexin V flow cytometry: adjunct to morphologic and cytogenetic diagnosis of myelodysplastic syndromes. Cytometry 46: 28-32.

Miagostovich MP, Nogueira RM, Cavalcanti SM, Marzochi KB, Schatzmayr HG 1993. Dengue epidemic in the state of Rio de Janeiro, Brazil: virological and epidemiological aspects. Rev Inst Med Trop Sao Paulo 35: 149-154.

Molica S, Mannella A, Dattilo A, Levato D, Iuliano F, Peta A, Consarino C, Magro S 1996. Differential expression of BCL-2 oncoprotein and Fas antigen on normal peripheral blood and leukemic bone marrow cells. A flow cytometric analysis. Haematologica 81: 302-309.

Navarro-Sanchez E, Altmeyer R, Amara A, Schwartz O, Fieschi F, Virelizier JL, Arenzana-Seisdedos F, Despres P 2003. Dendriticcell-specific ICAM3-grabbing non-integrin is essential for the productive infection of human dendritic cells by mosquito-cellderived dengue viruses. EMBO Rep 4: 723-728.

Neves-Souza PC, Azeredo EL, Zagne SM, Valls-de-Souza R, Reis SR, Cerqueira DI, Nogueira RM, Kubelka CF 2005. Inducible nitric oxide synthase (iNOS) expression in monocytes during acute dengue fever in patients and during in vitro infection. BMC Infect Dis 5: 64.

Nogueira RMR, de Araújo JMG, Schatzmayr HG 2007. Dengue viruses in Brazil, 1986-2006. Rev Panam Salud Publica 22: 358-363.

Oyaizu N, Pahwa S 1995. Role of apoptosis in HIV disease pathogenesis. J Clin Immunol 15: 217-231.

Park DR, Thomsen AR, Frevert CW, Pham U, Skerrett SJ, Kiener PA, Liles WC (2003). Fas (CD95) induces proinflammatory cytokine responses by human monocytes and monocyte-derivedmacrophages. J Immunol 170: 6209-6216.

Reis SR, Sampaio AL, Henriques MG, Gandini M, Azeredo EL, Kubelka CF 2007. An in vitro model for dengue virus infection that exhibits human monocyte infection, multiple cytokine production and dexamethasone immunomodulation. Mem Inst Oswaldo Cruz 102: 983-990.

Schmidt T, Karsunky H, Frass B, Baum W, Denzel A, Moroy T 2000. A novel protein (Fbf-1) that binds to CD95/APO-1/FAS and shows sequence similarity to trichohyalin and plectin. Biochim Biophys Acta 1493: 249-254.

Servet-Delprat C, Vidalain PO, Azocar O, Le Deist F, Fischer A, Rabourdin-Combe C 2000. Consequences of Fas-mediated human dendritic cell apoptosis induced by measles virus. $J$ Virol 74: 4387-4393.

Seth RB, Sun L, Chen ZJ 2006. Antiviral innate immunity pathways. Cell Res 16: 141-147.

Shao RG, Shimizu T, Pommier Y 1996. Brefeldin A is a potent inducer of apoptosis in human cancer cells independently of p53. Exp Cell Res 227: 190-196.

Strauss-Ayali D, Conrad SM, Mosser DM 2007. Monocyte subpopulations and their differentiation patterns during infection. J Leukoc Biol 82: 244-252.

Sydow FF, Santiago MA, Neves-Souza PC, Cerqueira DI, Gouvea AS, Lavatori MF, Bertho AL, Kubelka CF 2000. Comparison of dengue infection in human mononuclear leukocytes with mosquito C6/36 and mammalian Vero cells using flow cytometry to detect virus antigen. Mem Inst Oswaldo Cruz 95: 483-489.

Vermes I, Haanen C, Steffens-Nakken H, Reutelingsperger C 1995. A novel assay for apoptosis. Flow cytometric detection of phosphatidylserine expression on early apoptotic cells using fluorescein labelled Annexin V. J Immunol Methods 184: 39-51.

Wang WH, Gregori G, Hullinger RL, Andrisani OM 2004. Sustained activation of p38 mitogen-activated protein kinase and c-Jun N-terminal kinase pathways by hepatitis B virus X protein mediates apoptosis via induction of Fas/FasL and tumor necrosis factor (TNF) receptor 1/TNF-alpha expression. Mol Cell Biol 24: 10352-10365.

Wu SJ, Grouard-Vogel G, Sun W, Mascola JR, Brachtel E, Putvatana R, Louder MK, Filgueira L, Marovich MA, Wong HK, Blauvelt A, Murphy GS, Robb ML, Innes BL, Birx DL, Hayes CG, Frankel SS 2000. Human skin Langerhans cells are targets of dengue virus infection. Nat Med 6: 816-820.

Yu Q, Chow EM, Wong H, Gu J, Mandelboim O, Gray-Owen SD, Ostrowski MA 2006. CEACAM1 (CD66a) promotes human monocyte survival via a phosphatidylinositol 3-kinase- and AKT-dependent pathway. J Biol Chem 281: 39179-39193. 